> L'adolescence est souvent l'âge de l'expérimentation des substances psychoactives et parfois celui du passage à un usage plus régulier. À travers les résultats d'enquêtes en population générale menées en France, cet article fait le point sur les consommations des drogues des plus jeunes en mettant en évidence leurs spécificités par rapport à celles des adolescents plus âgés. II montre que les usages réguliers des différentes substances ne commencent en général guère avant 14 ans, mais que les adolescents qui se distinguent par la précocité de leur expérimentation présentent un risque nettement plus important de basculer vers un usage plus intensif ou problématique. À travers une présentation des limites méthodologiques des enquêtes utilisées, les auteurs discutent la nature du lien observé entre précocité de l'expérimentation et niveau d'usage : tout en rappelant l'indiscutable valeur pronostique de la précocité du premier usage pour prédire les usages problématiques futurs, ils montrent que son interprétation peut se révéler délicate dans les enquêtes épidémiologiques transversales. <

\section{Les usages \\ de drogues \\ des plus jeunes \\ adolescents}

1. Données épidémiologiques

François Beck, Emmanuelle Godeau,

Stéphane Legleye, Stanislas Spilka

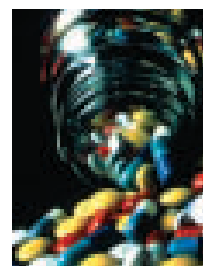

\author{
Département Observation \\ et analyse des comportements de santé, \\ INPES, 42, boulevard de la Libération, \\ 93203 Saint-Denis Cedex, France. \\ CESAMES, Centre de recherche \\ Psychotropes, Santé mentale, Société, \\ CNRS UMR 8136, Inserm U611, \\ Université Paris Descartes, Paris V \\ 45 , rue des Saints-Pères, \\ 75270 Paris Cedex 06, France. \\ francois.beck@inpes.sante.fr
}

lien entre précocité et niveau d'usage et les difficultés d'interprétation de la précocité comme un indicateur de gravité des usages futurs.

\section{Sources de données}

Trois enquêtes menées en France par questionnaire auto-administré et strictement anonyme auprès des adolescents ont été mobilisées. Health Behaviour in School-aged Children (HBSC), réalisée en 2002 dans 35 nations d'Europe et d'Amérique du Nord, interroge les élèves de 11,13 et 15 ans sur leurs comportements de santé [1]. Les questions relatives à la consommation de drogues illicites n'y sont pas posées aux adolescents de 11 et 13 ans, le questionnaire ne recueillant à ces âges que des données sur leurs usages d'alcool et de tabac. European school Survey Project on Alcohol and other Drugs (ESPAD), dont le dernier exercice publié a été mené en 2003, permet pour sa part d'observer les usages des jeunes gens scolarisés dans 35 pays d'Europe dont la France [2], en particulier ceux âgés de 12 à 16 ans qui se trouvent soumis à l'obligation scolaire. Enfin, l'Enquête sur la Santé et les Consommations lors de l'Appel de Préparation à la Défense (ESCAPAD) permet de faire le point sur les niveaux de consommation de substances psychoactives des jeunes de 17-18 ans et de présenter les évolutions récentes de ces pratiques à la fin de l'adolescence, en France métropolitaine et dans les départements et collectivités 


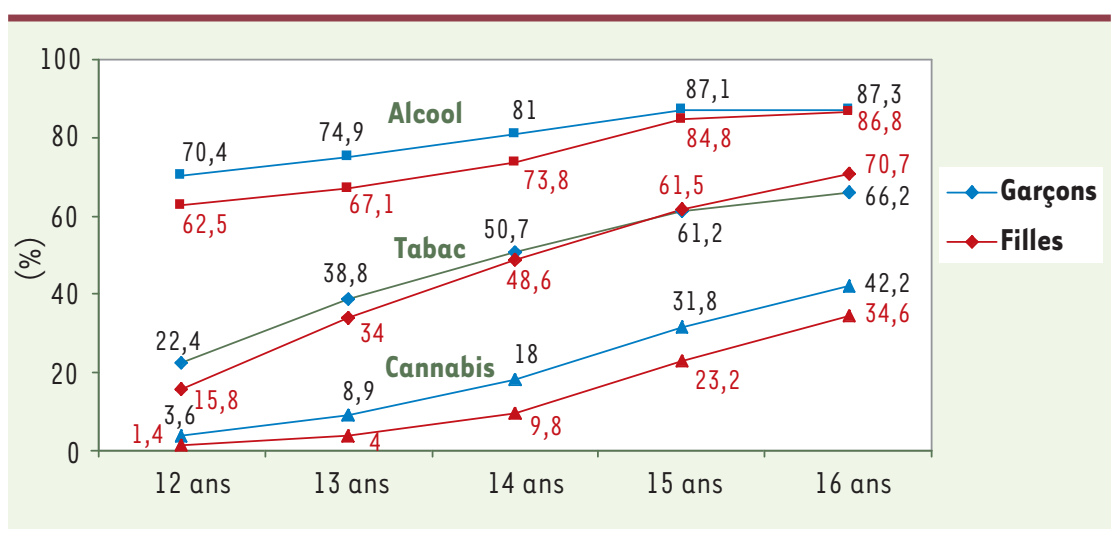

Figure 1. Expérimentations du tabac, du cannabis et de l'alcool: prévalences au cours de la vie par âge et par sexe. Source : ESPAD 03, Inserm, OFDT, MENRT.

d'Outre-mer [3]. Ces trois enquêtes documentent donc la diffusion des usages de drogues tout au long de l'adolescence, entre 11 et 18 ans. Dans cet article, les données concernant les élèves de 11 ans sont issues de l'enquête HBSC 2002, celles concernant les 12-16 ans de l'enquête ESPAD 2003 et celles concernant les jeunes de 17-18 ans de l'enquête ESCAPAD $2003^{1}$.

\section{Précocité et diffusion des usages}

Calendriers des expérimentations d'alcool, de tabac et de cannabis Si les niveaux d'expérimentation augmentent avec l'âge, les progressions des produits sont différentes (Figure 1). Pour le cannabis, les prévalences sont très faibles à 12 ans, croissent très vite jusqu'à 14 ans et progressent plus faiblement ensuite. Pour le tabac, les proportions d'expérimentateurs croissent vite de 12 à 14 ans puis le rythme de progression se fait de moins en moins soutenu et l'écart entre les sexes diminue. Enfin, contrairement aux autres produits, l'expérimentation de l'alcool est déjà généralisée à 12 ans : la progression est donc plus lente, notamment à partir de 14 ans, et se caractérise surtout par un rattrapage des filles. L'expérimentation de l'alcool a en effet généralement lieu dans le cadre familial sous le contrôle des parents; par contraste, celles de tabac et de cannabis sont plus transgressives, et ont souvent lieu à leur insu. Les significations de ces comportements sont donc très dissemblables, ce qui explique en partie les niveaux très inégaux des expérimentations à 12 ans, mais aussi leurs rythmes contrastés de progression (Figure 1).

\section{Expérimentations de substances illicites autres que le cannabis} Les niveaux d'expérimentation des autres drogues s'avèrent faibles, toujours inférieurs à $5 \%$, hormis pour les produits à inhaler (colles, solvants) et les champignons hallucinogènes. Quels que soient l'âge et le produit, l'expérimentation apparaît plus fréquente parmi les

${ }^{1}$ Précisons d'emblée que pour des raisons méthodologiques discutées plus loin, les données relatives aux plus jeunes sont rares. Ainsi, pour les 11 ans, seuls sont disponibles les niveaux d'expérimentation suivants : $14,4 \%$ des garçons et $9,7 \%$ des filles $(p<0,001)$ déclarent avoir déjà essayé de fumer du tabac et $18,4 \%$ des garçons et $7,8 \%$ des filles se déclarent consommateurs d'alcool au moment de l'enquête. L'article se concentrera donc essentiellement sur les $12-16$ ans. garçons (Tableau I). Si elle augmente avec l'âge pour des produits tels que les champignons hallucinogènes, l'ecstasy ou le LSD, pour les produits à inhaler elle stagne chez les garçons et régresse même légèrement chez les filles. Le cas des produits à inhaler apparaît tout à fait singulier : ils sont expérimentés très précocement mais leur usage n'est presque jamais prolongé à l'âge adulte. Cela est sans doute dû à la grande accessibilité de ces produits, souvent au domicile familial, au caractère plutôt peu social de leur usage ainsi qu'à la nature de leurs effets qui ne suscitent que rarement le désir de poursuivre l'usage. D'après les âges d'initiation déclarés par les élèves de 15 ans dans l'enquête HBSC 2002, $55 \%$ des élèves ayant déjà essayé les produits à inhaler l'auraient fait pour la première fois avant l'âge de 12 ans et seuls $7 \%$ à l'âge de 15 ans.

Dans le Tableau I, il convient de ne pas accorder une importance excessive à la précision des valeurs et aux écarts entre les différents âges, car pour la plupart ils ne sont pas significatifs. De plus, pour certaines substances, la validité de l'enquête déclarative auprès des plus jeunes est à mettre en question, une mécompréhension n'étant pas à exclure. De même, le pourcentage d'expérimentateurs d'amphétamines observé chez les plus jeunes est vraisemblablement lié au moins en partie aux traitements médicamenteux de l'hyperactivité. II faut retenir que dès l'âge de 14 ans, la plupart des drogues illicites autres que le cannabis ont été expérimentées par environ $2 \%$ des adolescents, mais que la majorité ne renouvelle pas l'expérience. Très peu sont donc véritablement concernés par leur consommation.

\section{Diffusion des usages réguliers}

d'alcool, de tabac et de cannabis

Les usages réguliers ${ }^{2}$ de tabac ou d'alcool apparaissent relativement rares avant 14 ans et celui de cannabis est exceptionnel avant l'âge de 15 ans. Ensuite, ils augmentent avec l'âge, le tabagisme quotidien devançant toutefois nettement la consommation régulière d'alcool et de cannabis (Figure 2). Ainsi, la proportion de fumeurs quotidiens de tabac passe de $1 \%$ à $11 \%$ entre 12 et 15 ans chez les garçons et de $0 \%$ à $14 \%$ chez les filles dans l'enquête ESPAD ( $0,6 \%$ de fumeurs quotidiens à 11 ans dans l'enquête HBSC, sans différence significative selon le sexe). On observe également une

${ }^{2}$ Usages réguliers dans cette population: usage au moins dix fois au cours des 30 derniers jours pour alcool et cannabis; quotidien pour le tabac. 


\begin{tabular}{|c|c|c|c|c|c|}
\hline Garçons & 12 ans* & 13 ans* & 14 ans & 15 ans & 16 ans \\
\hline Produits à inhaler & 7,5 & 9,7 & 11,3 & 11,6 & 11,6 \\
\hline Amphétamines & $\sim 0$ & 2,1 & 2,0 & 3,4 & 2,8 \\
\hline Ecstasy & $\sim 0$ & 2,0 & 1,7 & 3,9 & 3,5 \\
\hline Champignons hallucinogènes & - & - & 2,1 & 5,2 & 6,9 \\
\hline LSD & - & - & $\sim 0$ & $\sim 1$ & $\sim 1$ \\
\hline Cocaïne & - & - & 2,5 & 4,0 & 2,7 \\
\hline Héroïne & - & - & $\sim 1$ & 2,7 & 2,3 \\
\hline Filles & 12 ans* & 13 ans* & 14 ans & 15 ans & 16 ans \\
\hline Produits à inhaler & 6,5 & 6,8 & 8,4 & 12,0 & 9,7 \\
\hline Amphétamines & $\sim 0$ & $\sim 1$ & $\sim 1$ & 1,9 & 2,0 \\
\hline Ecstasy & $\sim 0$ & $\sim 1$ & $\sim 1$ & 2,9 & 3,0 \\
\hline Champignons hallucinogènes & - & - & $\sim 0$ & 1,8 & 2,8 \\
\hline LSD & - & - & $\sim 0$ & $\sim 1$ & $\sim 1$ \\
\hline Cocaïne & - & - & 2,2 & 2,6 & 2,5 \\
\hline Héroïne & - & - & $\sim 0$ & $\sim 1$ & $\sim 1$ \\
\hline
\end{tabular}

Tableau I. Expérimentation d'autres produits psychoactifs: prévalences au cours de la vie par sexe et par âge (en \%). Source : ESPAD 03 - INSERM - OFDT - MENRT. (*) Pour les élèves de 12 et 13 ans, le questionnaire était simplifié et certains produits supprimés.

tendance à la hausse avec l'âge pour l'alcool et le cannabis, notamment chez les garçons et dans une moindre mesure chez les jeunes filles. Les consommations quotidiennes d'alcool déclarées par les élèves de 11 ans restent rares ( $2,5 \%$ des garçons et $0,8 \%$ des filles), ces déclarations méritant à cet âge d'être interprétées avec précaution. Comme chez les plus âgés, les consommations d'alcool s'avèrent toujours plus fréquentes chez les garçons que chez les filles (Figure 2).

\section{Principales boissons alcooliques consommées}

Les types d'alcool consommés au cours de l'adolescence se modifient avec l'âge (Figure 3). À 11 ans, le cidre vient en tête $(48,9 \%$ de consommateurs au moins occasionnels ${ }^{3}$ ), suivi par le vin (y compris le champagne) $(38,1 \%)$ puis la bière $(14,8 \%)$. À cet âge, les spiritueux (dénommés «alcools forts 》 dans les enquêtes) s'avèrent très peu consommés $(5,5 \%)$ et pour tous les types d'alcool, les consommations occasionnelles sont prépondérantes. Ces consommations sont toujours plus répandues parmi les garçons, en

${ }^{3}$ Dans l'enquête HBSC, un usage occasionnel correspond à ceux qui déclarent boire, ne serait-ce que rarement. particulier celle de bière $(17,6 \%$ de garçons de 11 ans vs $9,5 \%$ de filles). Entre 12 et 15 ans, le cidre et le champagne sont donc les premières boissons alcooliques consommées, vraisemblablement dans un cadre familial et festif. Ensuite, entre 15 et 18 ans, leur consommation se stabilise, voire diminue et se trouve progressivement remplacée par la bière et les spiritueux. Ainsi, à 16 ans les spiritueux sont les boissons les plus consommées par les garçons, devant la bière. Parmi les filles le classement s'avère différent: si les alcools forts restent les premières boissons consommées, la bière se trouve devancée par le champagne.

Le vin se distingue nettement des autres boissons alcooliques, sa consommation progresse très lentement et reste relativement faible durant toute l'adolescence. Le cidre se singularise également en étant la seule boisson dont la consommation diminue avec l'âge dès le milieu de l'adolescence.

Notons qu'aucune des enquêtes évoquées ici ne posait la question des prémix ${ }^{4}$, or dans les premiers résultats de l'enquête ESCAPAD $2005^{5}$, il apparaît qu'à 17 ans, les bières et les prémix sont les boissons les plus populaires avec $57,1 \%$ et $48,1 \%$ de sujets à en avoir bu au cours des trente derniers jours, devant les spiritueux puis les champagnes et vins mousseux, les cocktails et le vin qui arrive en sixième position [4]. La forte présence des prémix dans cette hiérarchie invite à considérer avec attention les usages de ces produits et les stratégies de commercialisation et de promotion qui leurs sont attachées et qui visent notamment les plus jeunes. Ils ont en effet d'abord disparu en 1997 à la suite d'une taxe spécifique et sont réapparus en 2002 à la faveur d'un contournement de la réglementation (apparition de produits ayant une teneur en sucre plus faible).

La grande majorité des élèves de 11 ans n'a jamais connu d'ivresse $(95,2 \%)$, les filles étant plus nombreuses que les garçons à être dans ce cas de figure $(98,1 \%$ vs $92,2 \%, p<0,001)$. Néanmoins, dès $12-13$ ans, $12,0 \%$ des garçons et $6,3 \%$ des filles déclarent avoir déjà été ivres, ces chiffres atteignant respectivement $29,3 \%$ et $24,3 \%$ à $14-15$ ans et $51,0 \%$ et $47,0 \%$ à $16-17$ ans.

${ }^{4}$ Il s'agit de mélanges de boissons sucrées et d'alcool fort. Ces boissons, parfois appelées alcopops, titrent de $5^{\circ}$ (pour la majorité d'entre elles) à $8^{\circ}$ environ.

${ }^{5}$ Les catégories d'alcool présentées ici sont communes aux trois enquêtes, à l'exception du champagne qui, dans l'enquête HBSC, est inclus dans la même catégorie que le vin. 


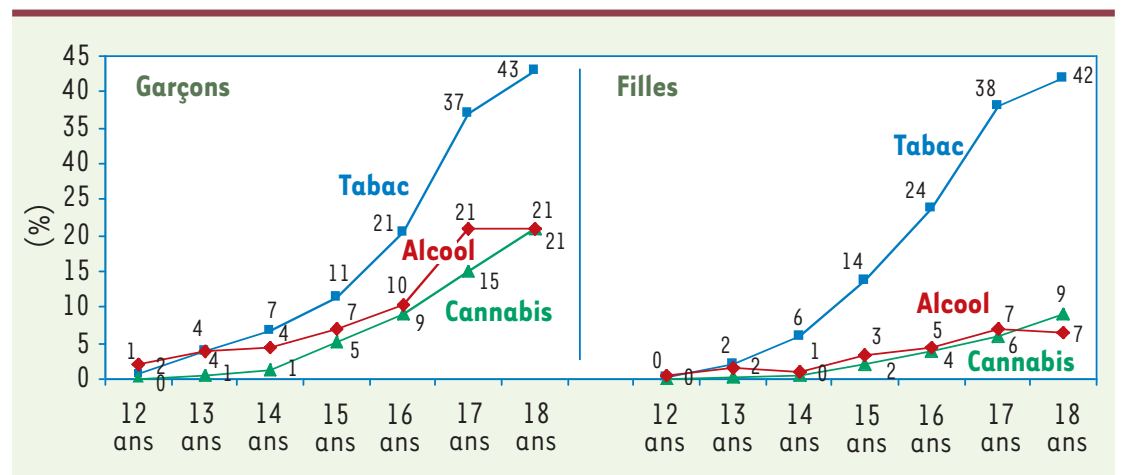

Figure 2. Usages réguliers de tabac, d'alcool et de cannabis, par âge et par sexe entre 12 et 18 ans en 2003. Source : ESPAD 2003, Inserm, OFDT, MJENR de 12 à 16 ans; ESCAPAD 2003 OFDT à 17 et 18 ans.

\section{L'interrogation des plus jeunes par les enquêtes en population générale : une question de méthode?}

L'interrogation des plus jeunes par les enquêtes en population générale présente quelques limites. En 1997, dans le Baromètre Santé Jeunes [5], les questions relatives à la consommation de drogues illicites n'étaient pas posées aux adolescents âgés de 12 à 14 ans. Cette précaution avait été prise à la suite de l'enquête pilote, menée en octobre 1997, au cours de laquelle les enquêteurs avaient relevé une fréquente gêne sur ce thème chez les plus jeunes, suscitant une hausse du taux de non-réponses. L'équipe de recherche avait également jugé qu'une telle interrogation, déconnectée de toute action de prévention et de toute possibilité d'échange approfondi sur la question, était de nature à perturber les moins renseignés parmi ceux qui étaient interrogés, ce qui est souvent le cas des plus jeunes. II est à noter qu'une même préoccupation a restreint, lors de l'enquête HBSC, les questions sur les drogues illicites aux élèves de 15 ans ou plus : pour les plus jeunes, la présence de ces questions aurait risqué de mettre en péril l'acceptation de l'enquête dans son ensemble, alors que celle-ci est une

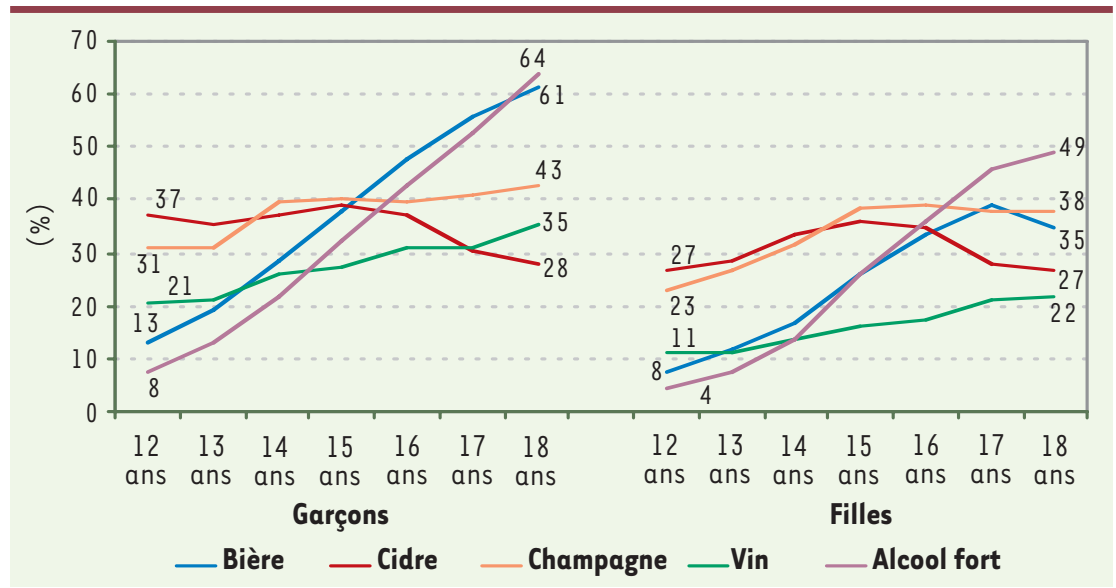

Figure 3. Type d'alcool consommé au moins une fois au cours des trente derniers jours en France par les adolescents selon le sexe et l'âge. Source : ESPAD 2003, Inserm, OFDT, MJENR. enquête généraliste bien acceptée même par les jeunes de 11 ans. Cette sensibilité est plus exacerbée dans certains pays, mais, dans un souci d'harmonisation, tous ont préféré retirer ces questions pour les 11 et les 13 ans.

Toutefois, dans le Baromètre santé 2000, la question de l'expérimentation de cannabis a été posée parmi les 12-14 ans. Au téléphone, les niveaux d'expérimentation ainsi déclarés sont assez bas (parmi les 12-14 ans seuls, $3,6 \%$ des garçons et $3,7 \%$ des filles ont répondu positivement) alors que les sollicitations s'avèrent déjà relativement importantes: à cet âge, $9,9 \%$ des garçons et $13,6 \%$ des filles déclarent s'être vus proposer du cannabis [6]. Ces niveaux peuvent être comparés à ceux obtenus de façon rétrospective (grâce à la question de l'âge au premier usage) par les jeunes interrogés dans ESCAPAD 2001 ; parmi eux, $6,8 \%$ des garçons et $3,4 \%$ des filles de 18 ans auraient expérimenté le cannabis dans la tranche d'âge 12-14 ans. Si pour les filles, les niveaux apparaissent très proches dans les deux enquêtes, les garçons sont deux fois plus nombreux à déclarer avoir déjà fumé du cannabis lorsque l'interrogation se fait de manière rétrospective, et ce malgré un effet génération négatif (les jeunes âgés de 18 ans lors de l'enquête ESCAPAD 2001 avaient 12 ans en 1995, époque à laquelle les niveaux d'usage du cannabis s'avéraient plus faibles qu'en 2000). Cet écart peut s'interpréter soit comme un rajeunissement de l'âge d'expérimentation du cannabis, soit comme un effet de la méthode d'interrogation, l'entretien téléphonique conduisant souvent à des déclarations d'usages de drogues plus faibles que le mode du questionnaire auto-administré [7], soit comme une résultante des deux effets. Alors que la connaissance de la précocité des expérimentations peut se révéler importante pour décrire la diffusion des usages dans la population et prédire son évolution future, les problèmes méthodologiques relatifs à l'interrogation des plus jeunes ne sont donc pas tout à fait résolus.

\section{Précocité et intensité de l'usage}

À partir d'une question posée sur l'âge au premier usage des différents produits, il est possible d'étudier le calendrier des consommations de façon relativement 
fiable si l'on se réfère aux études nord-américaines les plus récentes [8]. La précocité du premier usage de substance psychoactive en tant que facteur favorisant une persistance de cet usage $[9,10]$, voire une consommation problématique ou le passage à d'autres produits tels que la cocaïne ou l'héroïne à l'âge adulte [11], a été abondamment décrite par les épidémiologistes sur l'ensemble des substances psychoactives [12] et en particulier sur l'alcool [13-17]. Dans le cas du cannabis, cette influence d'une entrée précoce dans l'usage est parfois même jugée primordiale, pouvant compromettre l'apprentissage cognitif [18] et se trouver liée à une comorbidité psychiatrique [19] ou à des problèmes sociaux tels que le chômage, l'échec scolaire ou la délinquance [20]. À partir de l'enquête américaine du Substance Abuse and Mental Health Services Administration (SAMHSA), Gfroerer et al. ont ainsi montré que $62 \%$ des adultes de plus de 25 ans qui avaient été initiés au cannabis avant l'âge de 15 ans disent avoir consommé de la cocaïne au cours de leur vie, $9 \%$ de l'héroïne et $54 \%$ prendre fréquemment des médicaments psychotropes [21]. Ces chiffres s'avèrent nettement supérieurs à ceux observés sur l'ensemble de la population, respectivement $0,6 \%, 0,1 \%$ et $5,1 \%$.

Quelques travaux récents nuancent toutefois ces propos, évoquant notamment des biais possibles dans la mesure de la dépendance au cannabis [22]. Selon le rapport Roques sur la dangerosité des différentes drogues, si un environnement familial et socioculturel conflictuel dans l'enfance constitue un facteur de risque notable de dépendance à une substance psychoactive, celui-ci semble aggravé par la précocité de la première expérience [23]. Ces données tendent éga-

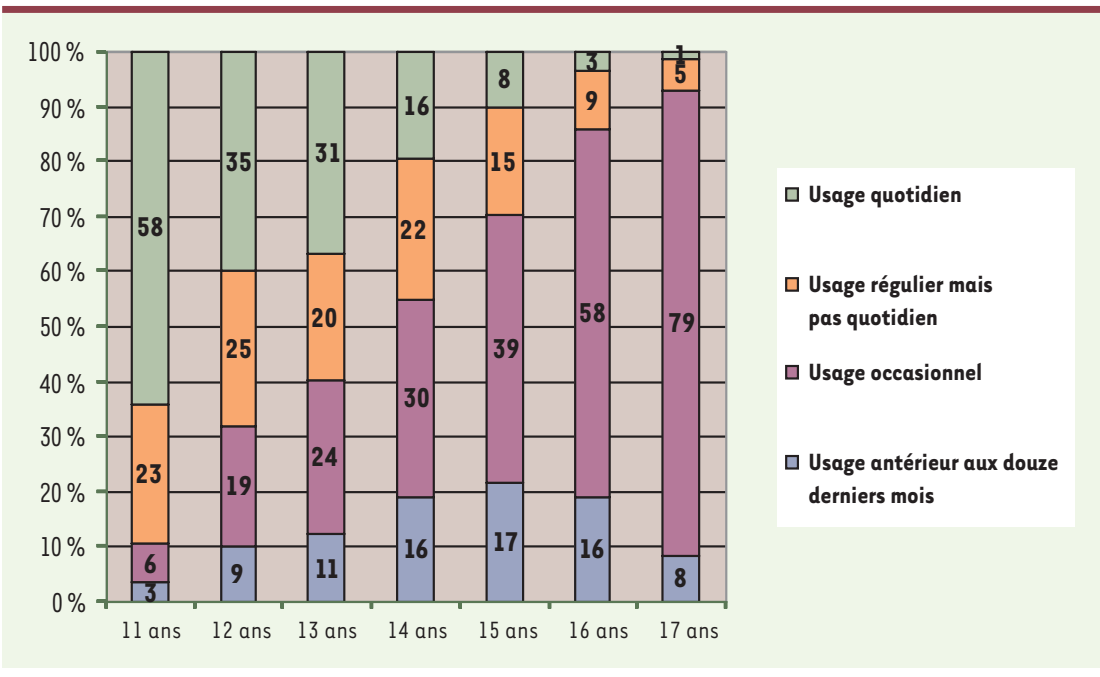

Figure 4. Type d'usage de cannabis à 17 ans en fonction de l'âge de premier usage. Source: ESCAPAD 2003, OFDT. lement à montrer la primauté de l'influence de la précocité du premier usage sur les autres variables envisagées (qui sont le statut scolaire, la proportion de consommateurs dans l'entourage, des signes anxiodépressifs, la consommation de soins et le statut socioprofessionnel des parents).

Il est possible d'explorer cette question à partir des données de l'enquête ESCAPAD 2003. II apparaît une évolution très marquée de la structure de répartition des types d'usage de cannabis avec l'accroissement de l'âge de premier usage de ce produit : la précocité est très liée à l'installation ultérieure dans une consommation importante de cannabis (Figure 4). Ainsi, $91 \%$ des jeunes de 17 ans qui ont fumé leur premier joint avant l'âge de 12 ans sont devenus des fumeurs réguliers de cannabis, et seuls $3 \%$ d'entre eux en ont abandonné la consommation.

Les acteurs de terrain de la toxicomanie s'accordent à souligner que l'engagement précoce dans une consommation est l'un des signes à observer avec attention. II semblerait que la précocité révèle fréquemment une situation de vulnérabilité psychosociale susceptible de conduire, au cours de l'adolescence, à des situations où l'usage devient problématique. Mais la force du lien entre précocité de l'expérimentation et maintien dans un usage régulier ne dispense pas d'une réflexion sur sa signification, dont on peut ici esquisser quelques traits.

En premier lieu, l'organisme des plus jeunes est plus fragile, en particulier leur cerveau, qui n'est pas tout à fait mature et peut en cela se révéler plus vulnérable. Sur le plan physiologique, si l'usage a été maintenu depuis la première expérience, la précocité reflète la chronicité de l'intoxication et aggrave souvent ses dangers, comme dans le cas de la consommation de tabac et des maladies qui lui sont liées. Sur le plan psychologique, de surcroît, il est plus difficile de renoncer à une habitude de consommation solidement installée qu'à un comportement récent. Ces éléments pourraient suffire à faire de la donnée de l'âge au premier usage un élément à surveiller sur un plan médical et sanitaire.

Néanmoins, d'un point de vue interprétatif, le fait que la précocité des expérimentations soit prédictive d'abus ultérieurs pourrait relever d'un certain attrait pour les substances psychoactives, qui inciterait à la fois à des usages plus variés, plus précoces et plus intenses. De plus, il est souvent difficile de distinguer l'effet propre de la précocité du premier usage de celui de la durée de l'usage (c'est-à-dire sa chronicité). En effet, il est raisonnable de penser qu'il faut un apprentissage, une habituation au produit pour le consommer de plus en plus fréquemment et éventuellement de façon de plus en plus problématique [24]. Par conséquent, la relation entre précocité et niveau d'usage est en partie aussi une relation entre durée d'usage et niveau d'usage. II est ainsi 
probable, même si les données ne permettent pas de le montrer, que la durée nécessaire au passage de l'expérimentation à l'usage quotidien de cannabis ne soit pas plus courte lorsque l'expérimentation a eu lieu à 11 ans que lorsqu'elle a eu lieu à 14 ans (pour des raisons pratiques et financières, le contraire semble même plus plausible). II n'est donc pas étonnant que, sans que cela lui ôte sa valeur pronostique, la précocité de l'usage n'entre pas dans les critères permettant de juger le caractère nocif, abusif ou dépendant d'une consommation de produit psychoactif selon le DSM-IV ou la CIM-10. Toutes choses égales par ailleurs, I'ancienneté de l'usage, et donc sa précocité, pourrait même être un synonyme d'une certaine stabilité de l'usage.

Par ailleurs, une expérimentation trop précoce pourrait être moins bien négociée dans la mesure où elle ne permettrait pas autant, par exemple, de s'appuyer sur l'expérience des pairs pour s'y préparer. Sans obérer cette hypothèse, la précocité pourrait aussi bien révéler la densité du réseau de pairs et l'intensité des relations amicales. Ainsi, dans l'enquête ESPAD 1999, les expérimentateurs les plus précoces apparaissent davantage entourés de pairs consommateurs : la proportion de jeunes de 15 ans estimant que la majorité de leurs amis boit de l'alcool décroît avec l'augmentation de l'âge d'expérimentation du premier verre d'alcool, passant, pour les alcools forts, de $40 \%$ pour une expérimentation à $11-12$ ans à $27 \%$ pour une expérimentation à $14-15$ ans $(p<0,01)$.

Pour ces raisons, les données transversales recueillies dans les enquêtes en population générale présentent d'importantes limites. Néanmoins, elles permettent, lorsqu'elles sont répétées, de documenter les changements intervenant dans la diffusion des produits psychoactifs dans la population. II est difficile de documenter une évolution des précocités au premier usage sur le long terme, cette question n'ayant été introduite pour la première fois en France qu'en 1999 dans l'enquête ESPAD. Dans le cas du cannabis, par exemple, ce recul ne permet pas de mettre en perspective la forte diffusion du cannabis et un net changement dans l'âge d'entrée dans sa consommation, même si une exploitation récente d'ESCAPAD permet d'esquisser une baisse significative depuis 2000, lorsqu'on interroge des adolescents de 17 ans: l'âge moyen déclaré au premier joint serait ainsi passé de 15,3 ans à 15,1 ans entre 2000 et 2005 [25].

\section{Conclusion}

Si les usages réguliers des différentes substances psychoactives ne commencent en général guère avant l'âge de 14 ans, il est notable que les adolescents qui se distinguent par la précocité de leur pratique constituent une population présentant une certaine vulnérabilité et un risque important de basculer vers un usage problématique. Sans pouvoir être assimilée à d'autres facteurs psychosociaux exogènes, la précocité des usages peut néanmoins servir de signal d'une situation à risque. Au-delà des risques strictement médicaux, la pratique atypique que constitue un usage précoce par rapport à la norme en vigueur dans son entourage prive en effet le jeune adolescent d'un certain nombre de facteurs de protection, tel que le contrôle par les pairs usagers susceptible de fixer des limites qui, si elles transgressent les normes des adultes par exem- ple, n'autorisent pas pour autant toutes les formes d'excès. Les programmes de prévention semblent prendre de plus en plus en compte cette précocité en ne refusant pas le dialogue sur les substances psychoactives dès la fin du primaire. Même s'il en existe d'autres, être un très jeune usager de drogues constitue donc un critère pronostic pertinent pour le repérage et la prévention éventuelle des usages problématiques avant leur manifestation effective. Or une telle démarche s'avère de plus en plus cruciale pour faire porter l'effort plus sur un évitement du basculement vers la toxicomanie que sur la sortie de cette dernière. Certes, du point de vue des risques de pathologies survenant à long terme, comme les cancers, la donnée de la durée d'usage, et donc de la précocité du premier usage revêt une grande importance. En revanche, il importe de ne pas la considérer comme le seul élément diagnostic dans la détermination du caractère problématique de l'usage. En effet, la réaction de l'expérimentateur lors des premières consommations est un élément tout aussi crucial. Une mauvaise expérience peut éventuellement dissuader l'expérimentateur de renouveler sa tentative, alors qu'au contraire une première consommation plaisante peut l'inciter à poursuivre. Le renoncement courant, avec l'avancée en âge, à des produits tels que les colles et solvants qui sont pourtant très accessibles, en offre une illustration. Le déplaisir, le plaisir et éventuellement l'apprentissage, solitaire ou en groupe, de la consommation, sont de puissants éléments qui conduisent l'individu à faire des choix, à commencer par celui de renouveler ou non la consommation de cette drogue. $\diamond$

\section{SUMMARY}

Drug consumptions by the young adolescents: epidemiological data

Adolescence is often the time of experimentation with psychoactive substances, sometimes leading to more regular use. This paper gives an update of drug consumptions by the young adolescents, from results of recent general population surveys in France, and focuses on the specificity of this consumption when compared to that of older adolescents. It shows that regular uses of such substances usually do not start before the age of 14 , but that early initiated adolescents show a higher risk of moving towards more intensive or problematic uses. Through presenting the limitations of such surveys, the authors discuss the nature of the link observed between early experimentation and level of use: while acknowledging the unquestionable prognostic value of early initiation to predict future problematic use, they show that its interpretation should be made with caution when based on such transversal epidemiological surveys. $\diamond$ 


\section{RÉFÉRENCES}

1. Godeau $\varepsilon$, Grandjean H, Navarro F. La santé des élèves de onze à quinze ans en France/2002. Saint-Denis : INPES, $2005: 286$ p.

2. Choquet $M$, Beck F, Hassler $C$, et al. Les substances psychoactives chez les collégiens et lycéens : consommations en 2003 et évolutions depuis dix ans. Tendances $2004 ; 35: 1-6$.

3. Beck F, Legleye S, Spilka S. Drogues à l'adolescence. Niveaux et contextes d'usage de substances psychoactives à 17-18 ans en France. ESCAPAD 2003. Saint-Denis : OFDT, $2004: 252$ p.

4. Beck F, Legleye S, Spilka S. Alcool, cannabis, tabac: contextes d'usages, prises de risque et tendances récentes; principaux résultats de l'enquête ESCAPAD 2005. Tendances $2006: 49: 1-4$.

5. Baudier F, Janvrin M-P, Arènes J. Baromètre santé jeunes 1997/1998. Vanves : Les Éditions du CFES, 1998 : 328 p.

6. Beck F. Usages de drogues illicites. Baromètre santé $2000 ; 1: 1-4$.

7. Beck F, Peretti-Watel P. Influence du mode de collecte sur les usages de drogues illicites déclarés par les 15-19 ans. Population 2001; $56: 963-86$.

8. Johnson TP, Mott JA. The reliability of self-reported age of onset of tobacco, alcohol and illicit drug use. Addiction 2001; 96 : 1187-98.

9. Robins LN. The natural history of adolescent drug use. Am J Publ Health $1984 ; 74: 656-7$.

10. Windle M. An alcohol involvement typology for adolescents: convergent validity and longitudinal stability. J Stud Alcohol 1996; $57: 627-37$.

11. Yamaguchi K, Kandel DB. Patterns of drug use from adolescence to young adulthood: sequence of progression. Am J Publ Health 1984 ; 74 : 668-72.

12. Kraus $L$. Is there a shift in age of first cannabis use towards younger ages? Data revisited in Germany, France, Greece and Spain. Ghent (Belgium) : $14^{\text {th }}$ International Conference of the European Society for Social Drug Research (ESSD), 2-4 octobre 2003.

13. De Wit DJ, Adlaf EM, Offord DR, Ogborne AC. Age at first alcohol use: a risk factor for the development of alcohol disorders. Am J Psychiatry $2000 ; 157: 745-50$.
14. Hawkins JD, Graham JW, Maguin $\varepsilon$, et al. Exploring the effects of age of alcohol use initiation and psychosocial risk factors on subsequent alcohol misuse. J Studies Alcohol 1997 ; $58: 280-90$.

15. Hingson R, Heeren T, Winter MR, Wechsler H. Early age of first drunkenness as a factor in college students' unplanned and unprotected sex attributable to drinking. Pediatrics 2003 ; $111: 34-41$.

16. Godeau $\varepsilon$, Vignes C, Ter Bogt T, et al. Consommation de cannabis par les élèves de 15 ans, données issues de l'enquête internationale HBSC/OMS dans 32 pays occidentaux. Alcoologie et Addictologie $2006 ; 28: 135-42$.

17. Kraus L, Bloomfield K, Augustin R, Reese A. Prevalence of alcohol use and the association between onset of use and alcohol-related problems in a general population sample in Germany. Addiction 2000 ; $95: 1389-401$.

18. Pope HG Jr, Gruber AJ, Hudson JI, et al. Early-onset cannabis use and cognitive deficits: what is the nature of the association? Drug Alcohol Dependence $2003 ; 69: 303-10$.

19. Armstrong TD, Costello $\varepsilon$ J. Community studies on adolescent substance use, abuse, or dependence and psychiatric comorbidity. J Consulting Clinical Psychology $2002 ; 70$ : 1224-39.

20. Fergusson DM, Horwood LJ. Early onset cannabis use and psychosocial adjustment in young adults. Addiction $1997 ; 92: 279-96$

21. Gfroerer JC, Wu LT, Penne MA. Initiation of marijuana use: trends, patterns, and implications. Bethesda: MD, DHHS, SAMHSA, OAS, $2002: 148 \mathrm{p}$.

22. Chen CY, Anthony JC. Possible age-associated bias in reporting of clinical features of drug dependence: epidemiological evidence on adolescent-onset marijuana use. Addiction 2003 ; $98: 71-82$.

23. Roques B. Problèmes posés par la dangerosité des «drogues ». Rapport au secrétariat d'État à la santé. Paris : Éditions Odile Jacob/La Documentation Française, 1998 : 316 p.

24. Becker HS (1963). Outsiders. Paris : Métaillé, 1985 : 280 p.

25. Legleye S, Beck F, Le Nezet 0, Spilka S. Drogues à l'adolescence en 2005. Niveaux, contextes d'usage et évolutions à 17 ans en France. Paris : OFDT, $2007: 70$ p.
$\%$

CEVTEE NATIONAL. DE LA KFCRE WCHE scIENTIRQ⿻上丨

dv 13 au 14 mars 2008 Le risque chimique : oonnsiseance et prévention niveau I a GIF SUR YVETRE Y 913

de 19 au 23 moi 2008 Les biopuces t thborle et pratique a GIF SUR YVETRE (9I)

de 19 au $23 \mathrm{~ms} 2008$ Caracterisation des proteines par spectrométrie de masse dans le contexte de la protécmique ¿ PARIS (75)

de 26 ay $30 \mathrm{~ms} 2008$ initiation theorique et experimentale aux techniques de base de la biologie moieculaire a ORSAY $(91)$

de 26 au $30 \mathrm{~ms} 2008$ Microecoples electronique et b force atomique sppliquees aux complexes ACN-Proteinea ¿ VLLEJUIF (94)

du 14 au 18 jamier 2008 Formation de lo Personne Competente en Radioprotection - Module theorique relatif aux principes de Is ¿ CLERWONT FEARAND (65) rasioprotection et a la rbgiementation en matibre de radieprotection dana le secteur "mbdical"

du 2 au 6 juin 2008 Formation de la Personne Competente en Radioprotection - Module pratique relatif a la detention ou la

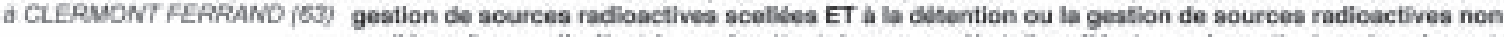
scellees, daposieils electriques émettant des rayons $X$ et dfaccélérateurs de particules, dans le secteur "medical"

Centre de ressouroes en formation

Un probieme de formation perticulier? Nhbshaz pess h nous ocreaser parmal a ressourcesedtorsogtif

- par wiphone au ot abs2 4496

Catalogue, programmes ef inscriations: CNRS Formation Entreprises Bät. 31 - Av. de la Terrasse 91198 Gir-sur-Yvette Cedex

Tel. : 0169824455 - Fax: 0169824489 Internet : hrtpulicnrsformation.cnra-git.fr 